\title{
Gender Differences in Lifestyle Factors Associated with Metabolic Syndrome and Preliminary Metabolic Syndrome in the General Population: The Watari Study
}

\author{
Tomomi Hattori $^{1}$, Satoshi Konno ${ }^{2}$ and Masanori Munakata ${ }^{1,2}$
}

\begin{abstract}
:
Objective In Japan, metabolic syndrome (MetS) and preliminary metabolic syndrome (preMetS) are more prevalent in men; however, it remains unclear whether the relationship between these metabolic disorders and lifestyle factors is similar between genders.

Methods We examined waist circumference, blood pressure, fasting blood, and various lifestyle factors in 3,166 individuals aged from 30-79 years of age from the Japanese general population. MetS was diagnosed on the basis of central obesity - assessed by waist circumference - plus two or more of the following cardiometabolic risks according to Japanese criteria: high blood pressure, hyperglycemia, and lipid abnormality. Central obesity plus one of the risks was defined as preMetS.

Results Men had a significantly higher prevalence of MetS $(23.3 \%$ vs. $8.7 \%, \mathrm{p}<0.001)$ and preMetS $(21.2 \%$ vs. $10.2 \%, \mathrm{p}<0.001)$ than women. An age-adjusted logistic regression analysis revealed that heavy drinkers were associated with an increased probability of MetS (odds ratio, 1.91: 95\% confidence interval, 1.29-2.83) and preMetS (1.69: 1.11-2.58); fast eaters were also related to preMetS (1.83: 1.33-2.55) and MetS (1.55: 1.12-2.15) in men. Lacking regular exercise was significantly associated with preMetS (1.38: 1.03-1.85), but not MetS. In women, preMetS was significantly associated with fast eaters and lacking regular exercise (1.44: 1.01-2.07 and 1.41: 1.02-1.96, respectively); a stepwise increase in each odds ratio (2.02: 1.40-2.91 and 1.47: 1.03-2.09, respectively) was also observed for MetS.

Conclusion The relationships between lifestyle factors and MetS or preMetS differed between men and women, which suggests the need for gender-specific lifestyle modification to effectively prevent MetS.
\end{abstract}

Key words: metabolic syndrome, preliminary metabolic syndrome, gender difference, lifestyle, Japanese general population

(Intern Med 56: 2253-2259, 2017)

(DOI: 10.2169/internalmedicine.8578-16)

\section{Introduction}

Cardio-metabolic risk factors, such as high blood pressure, hyperglycemia, and lipid abnormalities usually increase with age. Japanese criteria define metabolic syndrome (MetS) as the presence of central obesity and two or more of these cardio-metabolic risk factors (1). MetS is associated with an increased risk of diabetes and cardiovascular diseases (2-4). Moreover, it has been recently reported that even central obesity alone or with a single cardio-metabolic risk factor (preliminary metabolic syndrome [preMetS]) is associated with an increased risk of cardiovascular disease compared with non-obese healthy individuals (5); however, the absence of any significant relationship has also been reported (6). The incidence of diabetes is rapidly increasing in Japan $(7,8)$, which may be attributable to the aging population as well as MetS. Therefore, the early detection of MetS and preMetS and adequate lifestyle intervention are necessary to reduce the incidence of diabetes and cardiovascular disease, as well as to reduce medical costs. In order to implement this strategy at the national level, the Japanese gov-

${ }^{1}$ Research Center for Lifestyle-related Disease, Tohoku Rosai Hospital, Japan and ${ }^{2}$ Division of Hypertension, Tohoku Rosai Hospital, Japan Received: November 16, 2016; Accepted: January 11, 2017; Advance Publication by J-STAGE: August 10, 2017 Correspondence to Dr. Masanori Munakata, munakata@tohokuh.johas.go.jp 
ernment introduced a specified health examination system in 2008.

Abdominal visceral fat may accumulate due to an imbalance between energy intake and expenditure. Unhealthy lifestyle behaviors such as fast eating (9-11), skipping breakfast and having late-night meals (12), heavy drinking $(11,13,14)$, smoking $(11,14,15)$, and lacking regular exercise or a low physical activity $(11,15,16)$ have been associated with an increased risk of MetS. A Japanese government survey revealed that the prevalence of MetS and preMetS increases with age, and both are more predominant in men over all generations (17). However, only one study has examined the relationship between the all abovementioned lifestyles and MetS according to gender (18). Moreover, to our knowledge, no study has yet addressed the relationship between lifestyle factors and preMetS. To address these issues, we compared the relationships between MetS or preMetS and lifestyle factors between men and women in a Japanese rural population.

\section{Materials and Methods}

\section{Study design and population}

The Watari study is a prospective cohort study of the Japanese general population that has been conducted in the town of Watari, Miyagi Prefecture, Japan. The study examined whether albuminuria could predict cardiovascular events in the Japanese population. A detailed description of the study has been published elsewhere (19-21). The study protocol was approved by the ethics committee of Tohoku Rosai Hospital, and written informed consent was obtained from all participants.

The participants were individuals from Watari (ranging from 30-79 years of age, $42.6 \%$ men) who received an annual medical check-up in 2009.

\section{Measurements}

Information regarding past and current medical history and lifestyle factors (i.e., smoking status, regular exercise, the amount of alcohol intake per day, fast eating, late-night meal, fourth meal, and skipping breakfast) were examined using a part of standardized questionnaire of specific health examination (Appendix) (22). Medications for hypertension, diabetes, and dyslipidemia were assessed by questions 1-3. Participants who answered "yes" to question 8 were categorized as current smokers, while those who answered "no" to question 10 were categorized as lacking regular exercise. Being heavy drinkers (question 19) was defined as drinking two or more gou (gou=Japanese sake unit; 1 gou contains approximately $23 \mathrm{~g}$ of ethanol) for men and one or more gou for women. Participants who answered "faster" to question 14 were categorized as fast eaters. Participants who answered "yes" to questions 15-17 were categorized as commonly eating a late-night meal, a fourth meal, or skipping breakfast. Height, body weight, and waist circumference were measured by trained nurses. Body mass index was calculated as weight $(\mathrm{kg})$ divided by the height $(\mathrm{m})$ squared. After five minutes of seated rest, blood pressure was measured using a semiautomatic cuff-oscillometric sphygmomanometer (BX-10; Omron Colin, Kyoto, Japan). Blood samples were collected after an overnight fast and analyzed for low and high-density lipoprotein cholesterol, triglyceride, fasting blood sugar, and HbA1c (NGSP) levels. HbA1c level was determined by high-performance liquid chromatography (HLC723G7; Tosoh, Yamaguchi, Japan); the levels of the remaining biochemical markers were determined using a standard automatic analyzer 7700DD (Hitachi, Tokyo, Japan).

Following the recommendations from the Japanese committee for the Diagnostic Criteria of Metabolic Syndrome (1), we defined MetS as the presence of central obesity (waist circumference $=85 \mathrm{~cm}$ or more in men, $90 \mathrm{~cm}$ or more in women) as well as two or more risk factors among the following: lipid abnormality (triglycerides $\geq 150 \mathrm{mg} / \mathrm{dL}$ and/or high-density lipoprotein cholesterol $<40 \mathrm{mg} / \mathrm{dL}$ or use of medications for dyslipidemia), high blood pressure (systolic blood pressure $\geq 130 \mathrm{mmHg}$ and/or diastolic blood pressure $\geq 85 \mathrm{mmHg}$ or use of medications for hypertension), and hyperglycemia (fasting blood sugar $\geq 110 \mathrm{mg} / \mathrm{dL}$ or $\mathrm{HbA} 1 \mathrm{c} \geq 5.6 \%$ in case of non-fasting sampling or use of medications for diabetes). We defined preMetS as having central obesity plus one cardio-metabolic risk factor. Lastly, not having MetS meant not having central obesity or any of the cardio-metabolic risk factors. Dyslipidemia, hypertension, diabetes, and obesity were defined as low-density lipoprotein cholesterol $\geq 140 \mathrm{mg} / \mathrm{dL}$ and/or high-density lipoprotein cholesterol $<40 \mathrm{mg} / \mathrm{dL}$ or use of medications for dyslipidemia, systolic blood pressure $\geq 140 \mathrm{mmHg}$ and/or diastolic blood pressure $\geq 90 \mathrm{mmHg}$ or use of medications for hypertension, and $\mathrm{HbA} 1 \mathrm{c} \geq 6.5 \%$ or use of medications for diabetes, and body mass index $\geq 25 \mathrm{~kg} / \mathrm{m}^{2}$, respectively.

\section{Statistical analysis}

Of the 3,447 participants, we excluded 281 due to missing lifestyle $(n=11)$ and waist circumference $(n=270)$ data. Consequently, we used the data from 3,166 participants for the final analysis. The data were log-transformed in the case of skewed distributions. Group comparisons were performed using either Welch's t- or the chi-square tests. Men and women were classified by age: 30s, 40s, 50s, 60s, and 70s. Mantel-Haenszel tests for trends were used to assess the ptrends in cardio-metabolic risk factors and lifestyle factors over age groups. The relationship between MetS, preMetS or their components and each lifestyle factor were examined by a multiple logistic regression analysis with not having metabolic syndrome or each component as the control while adjusting for age. The data were presented as the means \pm standard deviation, medians (interquartile range), or percentages. A statistical analysis was performed using the IBM SPSS Statistics software program for Windows, version 20.0 (IBM, Armonk, USA). Statistical significance was defined as $\mathrm{p}<0.05$. 
Table 1. Clinical Characteristics.

\begin{tabular}{lccc}
\hline & $\begin{array}{c}\text { Men } \\
\mathrm{n}=1,280\end{array}$ & $\begin{array}{c}\text { Women } \\
\mathrm{n}=1,886\end{array}$ & $\mathrm{p}$ \\
\hline Age $(\mathrm{y})$ & $63.5 \pm 10.2$ & $60.5 \pm 12.0$ & $<0.001$ \\
WC $(\mathrm{cm})$ & $85.7 \pm 8.5$ & $82.9 \pm 9.6$ & $<0.001$ \\
BMI $\left(\mathrm{kg} / \mathrm{m}^{2}\right)$ & $23.8 \pm 3.0$ & $23.0 \pm 3.5$ & $<0.001$ \\
SBP $(\mathrm{mmHg})$ & $134.3 \pm 19.3$ & $130.0 \pm 20.0$ & $<0.001$ \\
DBP (mmHg) & $78.0 \pm 11.2$ & $72.8 \pm 10.8$ & $<0.001$ \\
TG $(\mathrm{mg} / \mathrm{dL})$ & $104(78,146)$ & $86(64,114)$ & $<0.001$ \\
HDL (mg/dL) & $57.6 \pm 15.2$ & $66.2 \pm 15.1$ & $<0.001$ \\
LDL (mg/dL) & $119.6 \pm 29.8$ & $125.9 \pm 31.0$ & $<0.001$ \\
FBS (mg/dL) & $97.3 \pm 21.7$ & $91.3 \pm 13.9$ & $<0.001$ \\
HbA1c (\%) & $6.0 \pm 0.8$ & $5.9 \pm 0.6$ & $<0.001$ \\
MetS (\%) & 23.3 & 8.7 & $<0.001$ \\
PreMetS (\%) & 21.2 & 10.2 & $<0.001$ \\
Central obesity (\%) & 51.8 & 22.3 & $<0.001$ \\
Lipid abnormality $(\%)$ & 35.7 & 27.0 & $<0.001$ \\
High blood pressure $(\%)$ & 65.9 & 56.1 & $<0.001$ \\
Hyperglycemia (\%) & 19.6 & 10.4 & $<0.001$ \\
Obesity (\%) & 31.4 & 26.1 & 0.001 \\
Dyslipidemia (\%) & 49.3 & 51.2 & 0.294 \\
Hypertension $(\%)$ & 53.5 & 41.6 & $<0.001$ \\
Diabetes (\%) & 16.4 & 9.9 & $<0.001$ \\
\hline Mea & & &
\end{tabular}

Means \pm SD or median (interquartile range). WC: waist circumference, BMI: body mass index, SBP: systolic blood pressure, DBP: diastolic blood pressure, TG: triglyceride, HDL: high-density lipoprotein cholesterol, LDL: low-density lipoprotein cholesterol, FBS: fasting blood sugar, HbAlc: hemoglobin A1c. MetS: metabolic syndrome, preMetS: preliminary MetS

\section{Results}

Table 1 shows the clinical characteristics of the men and women. Men were older and had generally worse cardiometabolic profiles compared to women, with the exception of dyslipidemia. MetS and preMetS were much more prevalent in men than in women (23.3 vs. $8.7 \%, \mathrm{p}<0.001$ and 21.2 vs. $10.2 \%, \mathrm{p}<0.001$, respectively) while the frequency of obesity was slightly higher in men than in women (31.4 vs. $26.1 \%, \mathrm{p}=0.001)$. Hypertension and diabetes were more prevalent in men than in women; however, the frequency of dyslipidemia did not differ between the two groups.

Table 2 compares the prevalence of cardio-metabolic risk factors between genders by ages. MetS and preMetS were more prevalent in men than in women in all age groups.

Accordingly the frequencies of central obesity, lipid abnormality, high blood pressure and hyperglycemia were higher in men in all age groups except for lipid abnormality in the 70s. The frequency of obesity was higher in men than in women in their 30s, but was similar between their 40s and 70s, except for those in their 60s. Hypertension was generally more prevalent in men than in women at all ages. Dyslipidemia was more prevalent in men in their 30 s and 40 s, but was more prevalent in women in their 60 s and 70 s. Diabetes was generally more prevalent in men than in women at all ages except for the 30s.
Table 3 shows the results of a multiple logistic regression analysis with MetS and preMetS as dependent variables. In men, being a heavy drinker was significantly associated with MetS [odds ratio (OR), 1.91: 95\% confidence interval (CI), 1.29-2.83) and preMetS (OR, 1.69: 95\% CI, 1.11-2.58). Being a fast eater was also related to preMetS (OR, 1.84: $95 \%$ CI, 1.33-2.55) and MetS (OR, 1.55: 95\%CI, 1.12-2.15). Lacking regular exercise was significantly associated with preMetS (OR, 1.38: 95\%CI, 1.03-1.85), but not with MetS. In women, preMetS was associated with significantly higher odds ratios for being a fast eater and lacking regular exercise (OR, 1.44: 95\%CI, 1.01-2.07 and 1.41: 1.02-1.96, respectively). A stepwise increase in each odds ratio (OR, 2.02: 95\%CI, 1.40-2.91 and 1.47: 1.03-2.09, respectively) was observed for MetS. The association between the components of MetS and unhealthy lifestyles was showed in the Supplementary material 1 . The relationship between central obesity and unhealthy lifestyles was quite similar to that between preMets and unhealthy lifestyles for both men and women.

Table 4 compares the frequency of unhealthy lifestyle factors between genders. Overall, the frequency of current smokers, fast eaters, heavy drinkers and late-night meals was higher in men than in women, while the lack of regular exercise was more prevalent in women. The frequency of a fourth meal and skipping breakfast did not differ between genders. 
Table 2. Frequency of MetS, PreMetS, Its Components and Cardiovascular Risks according to Age.

\begin{tabular}{|c|c|c|c|c|c|c|c|}
\hline & & \multicolumn{5}{|c|}{ Age categories } & \multirow[b]{2}{*}{$\mathrm{p}$ for trend } \\
\hline & & $30 \mathrm{~s}$ & $40 \mathrm{~s}$ & $50 \mathrm{~s}$ & $60 \mathrm{~s}$ & $70 \mathrm{~s}$ & \\
\hline \multirow[t]{2}{*}{ Number } & Men & 68 & 71 & 161 & 592 & 388 & \\
\hline & Women & 224 & 106 & 272 & 825 & 459 & \\
\hline \multirow[t]{2}{*}{ MetS (\%) } & Men & 11.8 & 18.3 & 29.2 & 22.3 & 25.3 & 0.073 \\
\hline & Women & $0.9^{*}$ & $3.8^{*}$ & $7.4^{*}$ & $9.2^{*}$ & $13.5^{*}$ & $<0.001$ \\
\hline \multirow[t]{2}{*}{ PreMetS (\%) } & Men & 16.2 & 16.9 & 16.1 & 23.0 & 22.2 & 0.068 \\
\hline & Women & $1.8^{*}$ & 11.3 & 10.7 & $10.7^{*}$ & $13.1^{\S}$ & $<0.001$ \\
\hline \multirow[t]{2}{*}{ Central obesity (\%) } & Men & 42.6 & 46.5 & 50.9 & 52.4 & 53.9 & 0.060 \\
\hline & Women & $6.7^{*}$ & $22.6^{\S}$ & $21.7 *$ & $23.8^{*}$ & $27.7 *$ & $<0.001$ \\
\hline \multirow[t]{2}{*}{ Lipid abnormality (\%) } & Men & 36.8 & 46.5 & 39.8 & 34.3 & 34.0 & 0.093 \\
\hline & Women & $1.8^{*}$ & $9.4^{*}$ & $18.0^{*}$ & 30.9 & $41.8^{\dagger}$ & $<0.001$ \\
\hline \multirow[t]{2}{*}{ High blood pressure $(\%)$} & Men & 23.5 & 36.6 & 62.7 & 69.3 & 74.7 & $<0.001$ \\
\hline & Women & 13.4 & 25.5 & $47.1^{\S}$ & $64.4^{\dagger}$ & 74.5 & $<0.001$ \\
\hline \multirow[t]{2}{*}{ Hyperglycemia (\%) } & Men & 4.4 & 12.7 & 18.6 & 21.5 & 21.1 & 0.007 \\
\hline & Women & 1.8 & $2.8^{\dagger}$ & $7.7 \S$ & $12.1^{*}$ & $14.8^{\dagger}$ & $<0.001$ \\
\hline \multirow[t]{2}{*}{ Obesity (\%) } & Men & 33.8 & 26.8 & 33.5 & 32.1 & 29.9 & 0.664 \\
\hline & Women & $10.3^{*}$ & 24.5 & 27.6 & $27.0^{\dagger}$ & 31.8 & $<0.001$ \\
\hline \multirow[t]{2}{*}{ Dyslipidemia (\%) } & Men & 45.6 & 52.1 & 55.9 & 48.3 & 48.2 & 0.549 \\
\hline & Women & $8.0^{*}$ & $23.6^{*}$ & 50.0 & $58.4^{*}$ & $66.4^{*}$ & $<0.001$ \\
\hline \multirow[t]{2}{*}{ Hypertension (\%) } & Men & 11.8 & 19.7 & 47.8 & 56.8 & 64.4 & $<0.001$ \\
\hline & Women & $3.1^{\S}$ & 15.1 & $25.7 *$ & $49.2^{\S}$ & 62.1 & $<0.001$ \\
\hline \multirow[t]{2}{*}{ Diabetes (\%) } & Men & 0.0 & 7.0 & 14.3 & 18.6 & 18.6 & 0.001 \\
\hline & Women & 0.4 & 2.8 & 8.1 & $10.8^{*}$ & 15.7 & $<0.001$ \\
\hline
\end{tabular}

${ }^{\dagger} \mathrm{p}<0.05,{ }^{{ }_{\mathrm{p}}}<0.01,{ }^{*} \mathrm{p}<0.001$ vs. men. MetS: metabolic syndrome, preMetS: preliminary MetS

Table 3. Association between MetS Or PreMetS and Unhealthy Lifestyles.

\begin{tabular}{|c|c|c|c|c|c|c|}
\hline & \multicolumn{3}{|c|}{ MetS } & \multicolumn{3}{|c|}{ preMetS } \\
\hline & OR & $95 \% \mathrm{CI}$ & $\mathrm{p}$ & OR & $95 \% \mathrm{CI}$ & $\mathrm{p}$ \\
\hline \multicolumn{7}{|l|}{ Men } \\
\hline Current smoker (vs. non-smoker) & 0.76 & $0.55-1.04$ & 0.084 & 0.77 & $0.55-1.06$ & 0.112 \\
\hline Lacking regular exercise (vs. presence of regular exercise) & 1.22 & $0.92-1.62$ & 0.172 & 1.38 & $1.03-1.85$ & 0.033 \\
\hline Fast eater (vs. normal and slow eater) & 1.55 & $1.12-2.15$ & 0.008 & 1.84 & $1.33-2.55$ & $<0.001$ \\
\hline Presence of late-night meal (vs. no) & 1.39 & $1.00-1.94$ & 0.053 & 1.08 & $0.76-1.55$ & 0.664 \\
\hline Presence of fourth meal (vs. no) & 1.00 & $0.58-1.73$ & 0.986 & 1.08 & $0.62-1.87$ & 0.789 \\
\hline Presence of skipping breakfast (vs. no) & 0.61 & $0.32-1.15$ & 0.125 & 0.66 & $0.35-1.24$ & 0.198 \\
\hline Heavy drinker (vs. non-or moderate drinker) & 1.91 & $1.29-2.83$ & 0.001 & 1.69 & $1.11-2.58$ & 0.015 \\
\hline \multicolumn{7}{|l|}{ Women } \\
\hline Current smoker (vs. non-smoker) & 0.98 & $0.39-2.45$ & 0.967 & 0.99 & $0.45-2.19$ & 0.978 \\
\hline Lacking regular exercise (vs. presence of regular exercise) & 1.47 & $1.03-2.09$ & 0.032 & 1.41 & $1.02-1.96$ & 0.040 \\
\hline Fast eater (vs. normal and slow eater) & 2.02 & $1.40-2.91$ & $<0.001$ & 1.44 & $1.01-2.07$ & 0.047 \\
\hline Presence of late-night meal (vs. no) & 1.22 & $0.76-1.98$ & 0.411 & 1.00 & $0.62-1.61$ & 0.988 \\
\hline Presence of fourth meal (vs. no) & 1.07 & $0.58-1.99$ & 0.823 & 0.75 & $0.39-1.42$ & 0.373 \\
\hline Presence of skipping breakfast (vs. no) & 1.05 & $0.49-2.24$ & 0.906 & 0.49 & $0.19-1.25$ & 0.134 \\
\hline Heavy drinker (vs. non-or moderate drinker) & 1.25 & $0.63-2.50$ & 0.518 & 1.74 & $1.00-3.04$ & 0.051 \\
\hline
\end{tabular}

MetS: metabolic syndrome, preMetS: preliminary MetS, OR: odds ratio, CI: confidence interval

\section{Discussion}

We observed in this Watari population that being a fast eater was significantly associated with the presence of MetS in both genders and that being a heavy drinker was significantly associated with the presence of MetS in men. The frequency of heavy drinkers was significantly higher in men than in women at all ages, while that of fast eaters was higher in men than in women in their 30s. These data sug- 
Table 4. Frequency of Unhealthy Lifestyles by Gender.

\begin{tabular}{|c|c|c|c|c|c|c|c|c|}
\hline & & \multirow{2}{*}{ Total } & \multicolumn{5}{|c|}{ Age categories } & \multirow[b]{2}{*}{$\mathrm{p}$ for trend } \\
\hline & & & $30 \mathrm{~s}$ & $40 \mathrm{~s}$ & $50 \mathrm{~s}$ & $60 \mathrm{~s}$ & $70 \mathrm{~s}$ & \\
\hline \multirow[t]{2}{*}{ Current smokers $(\%)$} & Men & 30.0 & 50.0 & 43.7 & 38.5 & 29.7 & 20.9 & $<0.001$ \\
\hline & Women & $5.4^{*}$ & $17.4^{*}$ & $17.0 *$ & $5.5^{*}$ & $2.9^{*}$ & $1.3^{*}$ & $<0.001$ \\
\hline \multirow[t]{2}{*}{ Lacking regular exercise $(\%)$} & Men & 56.4 & 69.1 & 73.2 & 73.3 & 53.9 & 47.9 & $<0.001$ \\
\hline & Women & $64.7 *$ & $87.5^{\S}$ & 82.1 & 71.3 & $60.6^{\dagger}$ & 52.9 & $<0.001$ \\
\hline \multirow[t]{2}{*}{ Fast eaters $(\%)$} & Men & 24.1 & 45.6 & 28.2 & 32.9 & 22.8 & 18.0 & $<0.001$ \\
\hline & Women & $20.8^{\dagger}$ & $20.1 *$ & 25.5 & 24.6 & 20.0 & 19.2 & 0.291 \\
\hline \multirow[t]{2}{*}{ Eating late-night meal (\%) } & Men & 20.8 & 22.1 & 22.5 & 26.7 & 21.2 & 17.3 & 0.066 \\
\hline & Women & $12.0^{*}$ & 14.7 & 17.9 & $11.8^{*}$ & $8.1^{*}$ & 16.6 & 0.604 \\
\hline \multirow[t]{2}{*}{ Eating fourth meal (\%) } & Men & 7.7 & 22.1 & 12.7 & 14.9 & 6.3 & 3.4 & $<0.001$ \\
\hline & Women & 8.7 & 17.9 & 17.0 & 12.9 & 5.5 & 5.7 & $<0.001$ \\
\hline \multirow[t]{2}{*}{ Skipping breakfast (\%) } & Men & 6.8 & 22.1 & 19.7 & 9.9 & 5.8 & 2.1 & $<0.001$ \\
\hline & Women & 5.3 & 12.1 & 12.3 & $4.4^{\dagger}$ & 4.0 & 3.3 & $<0.001$ \\
\hline \multirow[t]{2}{*}{ Heavy drinkers $(\%)$} & Men & 13.8 & 20.6 & 22.5 & 16.8 & 15.2 & 7.5 & $<0.001$ \\
\hline & Women & $7.4^{*}$ & 14.7 & 16.0 & $9.6^{\dagger}$ & $5.9 *$ & $3.1^{*}$ & $<0.001$ \\
\hline
\end{tabular}

${ }^{\dagger} \mathrm{p}<0.05,{ }^{\S} \mathrm{p}<0.01,{ }^{*} \mathrm{p}<0.001$ vs. men.

gest that gender differences in the prevalence of MetS may be explained in part by those dietary factors.

In women, substantially similar lifestyle factors; i.e. lacking regular exercise and being fast eaters were significantly associated with MetS and preMetS. Moreover, MetS was associated with a stepwise worsening of those lifestyle factors compared with preMetS. These data support the hypothesis that preMetS is an earlier stage of MetS and may develop to MetS by the continuation of an unhealthy lifestyle. Contrary to women, men demonstrated a different relationship between MetS, preMetS conditions, and lifestyle factors. MetS was associated with a higher odds ratio for heavy drinkers compared with preMetS, while the opposite was observed for fast eaters. Moreover, a lacking regular exercise was significantly associated with preMetS, but not with MetS. These data suggest that MetS does not simply develop from preMetS by the continuation of the same unhealthy lifestyle but may instead be associated with some qualitatively different lifestyle factors than preMetS. These data suggest the need for a gender-specific lifestyle strategy to effectively prevent MetS. An unhealthy lifestyle related to central obesity was quite similar to the risk related to preMets for both men and women suggesting that central obesity predisposes one to develop preMets in both genders.

Studies conducted in Japanese populations have shown that regular exercise lowers the risk for $\operatorname{MetS}(11,15,18)$. Moderate physical activity has also been shown to potentially decrease the risk of MetS and preMetS only in men (16). The reasons for the discrepancy between those previous reports and ours are unclear, but local occupational characteristics may be involved. The participants of this study were typically engaged in self-employed occupations, such as agriculture, fishing, forestry, and retail (19). Therefore, many men are likely to be engaged in hard physical activity, which may promote heavy drinking (23). In other words, lacking regular exercise does not necessarily result in low physical activity in this male MetS population. Additional data on regular activity as well as physical activity related to occupation are necessary to clarify this point.

The frequency of unhealthy lifestyle behaviors generally decreased with age in both sexes (Table 4). This finding suggests that the age-related increases in the frequencies of MetS and preMetS were chiefly attributable to non-lifestyle factors that develop with age. These may include age-related changes in endocrine and metabolic functions as well as the progression of sarcopenia $(24,25)$. In other words, only a subtle worsening of lifestyle could induce MetS in older generations. Conversely, MetS in young generations was largely attributable to lifestyle factors such as fast eating and heavy drinking seen in this male population.

In this study, the prevalence of MetS in men and women was $23.3 \%$ and $8.7 \%$, respectively; this is similar to data from the 2007 National Nutrition Survey, which reported a prevalence of $22.5 \%$ and $7.3 \%$ in men and women, respectively (17). The much lower prevalence of MetS in women than in men may be largely attributable to the unique Japanese diagnostic criteria in which the cut-off values for central obesity are waist circumferences of $85 \mathrm{~cm}$ and $90 \mathrm{~cm}$ for men and women, respectively. The International Diabetes Federation (IDF) criteria include cut-off values of $90 \mathrm{~cm}$ for men and $80 \mathrm{~cm}$ for women. A recent meta-analysis of 226,653 Chinese people examined by IDF criteria reported a pooled MetS prevalence of 24.5\% (95\% CI: 22.0$26.9 \%$ ) (26). By sex, the prevalence was $19.2 \%$ (95\%CI: $16.9-21.6 \%$ ) in men and $27.0 \%$ (95\% CI: $23.5-30.5 \%)$ in women. Thus, the prevalence of MetS is markedly influenced by the diagnostic criteria. The current Japanese criteria could underestimate cardiovascular risks in women. In fact, the prevalence of dyslipidemia was similar between men and women. Moreover, the differences in the prevalence of obesity, hypertension, and diabetes were much smaller than the difference in the prevalence of MetS. Re- 
cent reports have suggested that the cut-off value for central obesity for Japanese women should be $80 \mathrm{~cm}(27,28)$, the same as the value recommended by the IDF.

This study is associated with several limitations. First, the number of male participants in their 30 s and 40s was small; therefore, data from those groups may be biased. Second, the Watari town is a rural area of Japan and so the results of this study may not apply to urban populations. Third, we had no information on the amount of meal intake, which is a critical factor for the development of MetS (11). A lack of information on total physical activity including occupation was also another limitation, as noted above. Finally, the cross-sectional study design does not ensure that the significant lifestyle factors related to MetS identified in this study really increase the risk of incident MetS. Further prospective observational studies are therefore necessary to clarify this point.

\section{Conclusion}

Based on current Japanese criteria, the frequency of MetS and preMetS was more than three times higher in men than in women in a general rural Japanese population. Moreover, the relationship between lifestyles and MetS or preMetS differed between genders. In women, preMetS appeared to be an earlier stage of MetS; however, MetS was associated with qualitatively different lifestyle factors than preMetS in men. Our data suggest the need for a gender-specific lifestyle strategy to effectively prevent MetS.

The authors state that they have no Conflict of Interest (COI).

\section{Financial support}

This study was supported by the grant from the Japan Labor, Health, and Welfare Organization (Research Grant on the Lifestyle-related Disease).

\section{Acknowledgement}

The authors thank Professor Masayuki Tatemichi for his advice on the data analysis.

\section{References}

1. Definition and the diagnostic, standard for metabolic syndrome. Committee to Evaluate Diagnostic Standards for Metabolic Syndrome. Nihon Naika Gakkai Zasshi (J Jpn Soc Int Med) 94: 794809, 2005 (in Japanese).

2. Iso H, Sato S, Kitamura A, et al. Metabolic syndrome and the risk of ischemic heart disease and stroke among Japanese men and women. Stroke 38: 1744-1751, 2007.

3. Kokubo Y, Okamura T, Yoshimasa Y, et al. Impact of metabolic syndrome components on the incidence of cardiovascular disease in a general urban Japanese population: the Suita study. Hypertens Res 31: 2027-2035, 2008.

4. Ballantyne CM, Hoogeveen RC, McNeill AM, et al. Metabolic syndrome risk for cardiovascular disease and diabetes in the ARIC study. Int Obes 32 (Suppl 2): S21-S24, 2008.

5. Keihani S, Hosseinpanah F, Barzin M, Serahati S, Doustmohamadian S, Azizi F. Abdominal obesity phenotypes and risk of cardiovascular disease in a decade of follow-up: the Tehran lipid and glucose study. Atherosclerosis 238: 256-263, 2015.

6. Chei CL, Yamagishi K, Tanigawa $T$, et al. Metabolic syndrome and the risk of ischemic heart disease and stroke among middleaged Japanese. Hypertens Res 31: 1887-1894, 2008.

7. Mukai N, Doi Y, Ninomiya T, et al. Trends in the prevalence of type 2 diabetes and prediabetes in community-dwelling Japanese subjects: the Hisayama study. J Diabetes Investig 23: 162-169, 2014.

8. Ministry of Health, Labour and Welfare. The National Health and Nutrition Examination Survey in 2013 [Internet]. [cited 2016 Aug. 1]. Available from: http://www.mhlw.go.jp/bunya/kenkou/eiyou/dl/h 25-houkoku-03.pdf

9. Zhu B, Haruyama Y, Muto T, Yamazaki T. Association between eating speed and metabolic syndrome in a three-year populationbased cohort study. J Epidemiol 25: 332-336, 2015.

10. Nagahama S, Kurotani K, Pham NM, et al. Self-reported eating rate and metabolic syndrome in Japanese people: cross-sectional study. BMJ open 4: e005241, 2014.

11. Urashima M, Wada T, Fukumoto T, et al. Prevalence of metabolic syndrome in a 22892 Japanese population and its association with life style. JMAJ 48: 441-450, 2005.

12. Kutsuma A, Nakajima K, Suwa K. Potential association between breakfast skipping and concomitant late-night-dinner eating with metabolic syndrome and proteinuria in the Japanese population. Scientifica 2014: 253581, 2014.

13. Hirakawa M, Arase Y, Amakawa K, et al. Relationship between alcohol intake and risk factors for metabolic syndrome in men. Intern Med 54: 2139-2145, 2015.

14. Nakashita Y, Nakamura M, Kitamura A, Kiyama M, Ishikawa Y, Mikami H. Relationships of cigarette smoking and alcohol consumption to metabolic syndrome in Japanese men. J Epidemiol 20: 391-397, 2010.

15. Li Y, Yatsuya H, Iso $\mathrm{H}$, Tamakoshi $\mathrm{K}$, Toyoshima $\mathrm{H}$. Incidence of metabolic syndrome according to combinations of lifestyle factors among middle-aged Japanese male workers. Prev Med 2010 51: 118-122, 2010.

16. Kim J, Tanabe K, Yokoyama N, Zempo H, Kuno S. Association between physical activity and metabolic syndrome in middle-aged Japanese: a cross-sectional study. BMC public health 11: 624, 2011.

17. Ministry of Health, Labour and Welfare. The National Health and Nutrition Examination Survey in 2007 [Internet]. [cited 2008 Aug. 1]. Available from: http://www.mhlw.go.jp/bunya/kenkou/eiyou09/d 1/01-kekka.pdf

18. Ashizawa E, Katano S, Harada A, et al. Exploring the link between standard lifestyle questionnaires administered during specific medical check-ups and incidence of metabolic syndrome in Chiba Prefecture. Nihon Koshu Eisei Zasshi (Jpn J Public Health) 61: 176-185, 2014 (in Japanese).

19. Munakata $M$, Konno $S$, Ohshima $M$, Ikeda $T$, Miura $Y$, Ito S. High-normal blood pressure is associated with microalbuminuria in the general population: the Watari study. Hypertens Res 34: 1135-1140, 2011.

20. Konno S, Hozawa A, Miura Y, Ito S, Munakata M. High-normal diastolic blood pressure is a risk for development of microalbuminuria in the general population: the Watari study. J Hypertens 31: 798-804, 2013.

21. Konno S, Munakata M. Moderately increased albuminuria is an independent risk factor of cardiovascular events in the general Japanese population under 75 years of age: the Watari study. PLoS One 10: e0123893, 2015.

22. Shiga Federation of National Health Insurance Organization. Questionnaire for specific medical checkup [Internet]. [cited 2016 Aug. 1]. Available from: http://www.shigakokuho.or.jp/contents_de tail.php?co=kak\&frmId=60 
23. Cheng WJ, Cheng Y, Huang MC, Chen CJ. Alcohol dependence, consumption of alcoholic energy drinks and associated work characteristics in the Taiwan working population. Alcohol Alcohol 47: 372-379, 2012.

24. Guarner-Lans V, Rubio-Ruiz ME, Perez-Torres I, Banos de, MacCarthy G. Relation of aging and sex hormones to metabolic syndrome and cardiovascular disease. Exp Gerontol 46: 517-523, 2011.

25. Moon SS. Low skeletal muscle mass is associated with insulin resistance, diabetes, and metabolic syndrome in the Korean population: the Korea National Health and Nutrition Examination Survey (KNHANES) 2009-2010. Endocr J 61: 61-70, 2014.

26. Li R, Li W, Lun $\mathrm{Z}$, et al. Prevalence of metabolic syndrome in Mainland China: a meta-analysis of published studies. BMC pub- lic health 16: 296, 2016.

27. Doi Y, Ninomiya T, Hata J, et al. Proposed criteria for metabolic syndrome in Japanese based on prospective evidence: the Hisayama study. Stroke 40: 1187-1194, 2009.

28. Hu H, Kurotani K, Sasaki N, et al. Optimal waist circumference cut-off points and ability of different metabolic syndrome criteria for predicting diabetes in Japanese men and women: Japan Epidemiology Collaboration on Occupational Health Study. BMC public health 16: 220, 2016.

The Internal Medicine is an Open Access article distributed under the Creative Commons Attribution-NonCommercial-NoDerivatives 4.0 International License. To view the details of this license, please visit (https://creativecommons.org/licenses/ by-nc-nd/4.0/).

(C) 2017 The Japanese Society of Internal Medicine Intern Med 56: 2253-2259, 2017 